\title{
Kinetics, Dynamics and Energy of Solid on the Example of a Tool Fixed Flexibly: Part 2 - Dynamics
}

\author{
Zdzisław Pluta, Tadeusz Hryniewicz* \\ Koszalin University of Technology, Raclawicka 15-17, 75-620 Koszalin, Poland \\ *E-mail address: Tadeusz.Hryniewicz@tu.koszalin.pl
}

\begin{abstract}
This work is a continuation of the problems of kinetics, dynamics, and energy of solid on the example of a tool fixed flexibly under cutting. The work consists with three parts. Part 1 covered the kinetics of the system. Present work is devoted to the dynamics of a tool in the machining space-time. Dynamic characteristics of the whole machining system are considered by determining forces being the measures of particular reasons for material cutting. The reasons are the following forces: gravity, inertia, and repel. In the last Part 3 of the work, a special attention is to be paid to the work description and the energy notion.
\end{abstract}

Keywords: Tool; Dynamics; Machining space-time; Potential field; Potential; Mechanics

\section{INTRODUCTION}

For the introduction, the Authors' works [1,2] are referred to, concerning an adequate approach to cutting by the tool fixed flexibly. Specifically in [2] the third kinetic link, that is jerk as the derivative of acceleration with respect to time, was considered. The following two parts of the work are aiming at the dynamics and energy of the tool. To present a complexity of the problem, in these characteristics of the system, a cutter fixed together with an elastic element is assumed for consideration. Therefore the description of dynamic behaviour of the tool with the state energetic characteristics is to be presented.

A closer explanation of the time-effect notion will be provided in the context of tool dynamics [2-8]. A new method of solving the dynamics of the considered solid, with the solid being cutting tool fixed flexibly, is to be given. The source now will not be the equation of equilibrium of forces (fictional and real ones) but the equation of the path length of the tool edge.

\section{DYNAMICS OF SOLID/TOOL IN THE MACHINING SPACE-TIME}

Dynamics of a solid, here the tool fixed flexibly, is such its characteristics [1] in the variable motion where the body mass $m$ is taken into consideration. This mass, as given in Part 1 [2], equals $2.82 \mathrm{~kg}$.

By multiplying consecutive kinetic magnitudes: $v_{z}-$ velocity, $p_{z}-$ acceleration, $i_{z}-$ jerk, by the mass $m$, one obtains as follows:

- momentum $P_{z}$, i.e. 


$$
P_{z}=m \cdot v_{z}
$$

- inertia force $B_{z}$, then

$$
B_{z}=m \cdot p_{z}
$$

- and time-effect $\Pi_{z}$, then

$$
\Pi_{z}=m \cdot i_{z}
$$

Developing these dependences, by substituting the function records expressed by formulae (see Part 1: (1.7), (1.11), (1.14) [1]), one obtains

$$
\begin{gathered}
P_{z}=m \cdot v_{z}=m \frac{2 z^{*}}{T_{o}} e^{-\frac{t}{T_{o}}}=m v_{1}^{0} e^{-\frac{t}{T_{o}}}=P_{1}^{0} e^{-\frac{t}{T_{o}}} \\
B_{z}=m \cdot p_{z}=m \frac{2 z^{*}}{\left(T_{o}\right)^{2}} e^{-\frac{t}{T_{o}}}=m \frac{v_{1}^{0}}{T_{o}} e^{-\frac{t}{T_{o}}}=m p_{1}^{0} e^{-\frac{t}{T_{o}}}=B_{1}^{0} e^{-\frac{t}{T_{o}}} \\
\Pi_{z}=m \cdot i_{z}=m \frac{2 z^{*}}{\left(T_{o}\right)^{3}} e^{-\frac{t}{T_{o}}}=m \frac{v_{1}^{0}}{\left(T_{o}\right)^{2}} e^{-\frac{t}{T_{o}}}=m \frac{p_{1}^{0}}{T_{o}} e^{-\frac{t}{T_{o}}}=m \cdot i_{1}^{0} e^{-\frac{t}{T_{o}}}=\Pi_{1}^{0} e^{-\frac{t}{T_{o}}}
\end{gathered}
$$

where symbols $P_{1}^{0}, B_{1}^{0}, \Pi_{1}^{0}$ denote the initial momentum, initial inertia force, and initial time-effect, respectively, in the point 1 (Part 1: see Fig. 2 [2]). All these magnitudes $\left(P_{z}, B_{z}, \Pi_{z}\right)$ may be also expressed as the function of coordinate $x$, by regarding in formulae (2.4), (2.5), (2.6) the time $t$, determined by the dependence (1.2 [2]). Therefore

$$
\begin{gathered}
P_{z}=m \frac{2 z^{*}}{T_{o}} e^{-\frac{x}{v_{m} T_{o}}}=m v_{1}^{0} e^{-\frac{x}{v_{m} T_{o}}}=P_{1}^{0} e^{-\frac{x}{v_{m} T_{o}}} \\
B_{z}=m \frac{2 z^{*}}{\left(T_{o}\right)^{2}} e^{-\frac{x}{v_{m} T_{o}}}=m \frac{v_{1}^{0}}{T_{o}} e^{-\frac{x}{v_{m} T_{o}}}=m p_{1}^{0} e^{-\frac{x}{v_{m} T_{o}}}=B_{1}^{0} e^{-\frac{x}{v_{m} T_{o}}} \\
\Pi_{z}=m \frac{2 z^{*}}{\left(T_{o}\right)^{3}} e^{-\frac{x}{v_{m} T_{o}}}=m \frac{v_{1}^{0}}{\left(T_{o}\right)^{2}} e^{-\frac{x}{v_{m} T_{o}}}=m \frac{p_{1}^{0}}{T_{o}} e^{-\frac{x}{v_{m} T_{o}}}=m i_{1}^{0} e^{-\frac{x}{v_{m} T_{o}}}=\Pi_{1}^{0} e^{-\frac{x}{v_{m} T_{o}}}
\end{gathered}
$$

Next, regarding that the product $v_{m} \cdot T_{o}$ is the path constant $X_{o}$, as presented by formula (2.9), one obtains the following configurations of the higher given three magnitudes

$$
\begin{gathered}
P_{z}=m \frac{2 z^{*}}{T_{o}} e^{-\frac{x}{X_{o}}}=m v_{1}^{0} e^{-\frac{x}{X_{o}}}=P_{1}^{0} e^{-\frac{x}{X_{o}}} \\
B_{z}=m \frac{2 z^{*}}{\left(T_{o}\right)^{2}} e^{-\frac{x}{X_{o}}}=m \frac{v_{1}^{0}}{T_{o}} e^{-\frac{x}{X_{o}}}=m p_{1}^{0} e^{-\frac{x}{X_{o}}}=B_{1}^{0} e^{-\frac{x}{X_{o}}}
\end{gathered}
$$




$$
\Pi_{z}=m \frac{2 z^{*}}{\left(T_{o}\right)^{3}} e^{-\frac{x}{X_{o}}}=m \frac{v_{1}^{0}}{\left(T_{o}\right)^{2}} e^{-\frac{x}{X_{o}}}=m \frac{p_{1}^{0}}{T_{o}} e^{-\frac{x}{X_{o}}}=m i_{1}^{0} e^{-\frac{x}{X_{o}}}=\Pi_{1}^{0} e^{-\frac{x}{X_{o}}}
$$

After considering the earlier calculated values of $T_{o}$ and $X_{o}$, so $69.823 \mathrm{~s}$ and $130.57 \cdot 10^{-3} \mathrm{~m}$, respectively, as well as the mass value $m$ of the tool, i.e. $2.82 \mathrm{~kg}[1,2]$, one obtains

$$
\begin{aligned}
& P_{z}=m \frac{2 z^{*}}{T_{o}} e^{-\frac{x}{X_{o}}}=m v_{1}^{0} e^{-\frac{x}{X_{o}}}=p_{1}^{0} e^{-\frac{x}{X_{o}}}= \\
& =2.82 \frac{2 \cdot 7.835 \cdot 10^{-3}}{69.823} e^{-\frac{x}{130.57 \cdot 10^{-3}}}=2.82 \cdot 0.224 \cdot 10^{-3} e^{-\frac{x}{130.57 \cdot 10^{-3}}}= \\
& =6.32 \cdot 10^{-4} e^{-\frac{x}{130.57 \cdot 10^{-3}}} \\
& B_{z}=m \frac{2 z^{*}}{\left(T_{o}\right)^{2}} e^{-\frac{x}{X_{o}}}=m \frac{v_{1}^{0}}{T_{o}} e^{-\frac{x}{X_{o}}}=m p_{1}^{0} e^{-\frac{x}{X_{o}}}=B_{1}^{0} e^{-\frac{x}{X_{o}}}= \\
& =2.82 \frac{2 \cdot 7.835 \cdot 10^{-3}}{(69.823)^{2}} e^{-\frac{x}{130.57 \cdot 10^{-3}}}=2.82 \frac{0.224 \cdot 10^{-3}}{69.823} e^{-\frac{x}{130.57 \cdot 10^{-3}}}= \\
& =2.82 \cdot 3.208 \cdot 10^{-6} e^{-\frac{x}{130.57 \cdot 10^{-3}}}=9.05 \cdot 10^{-6} e^{-\frac{x}{130.57 \cdot 10^{-3}}} \\
& \Pi_{z}=m \frac{2 z^{*}}{\left(T_{o}\right)^{3}} e^{-\frac{x}{X_{o}}}=m \frac{v_{1}^{0}}{\left(T_{o}\right)^{2}} e^{-\frac{x}{X_{o}}}=m \frac{p_{1}^{0}}{T_{o}} e^{-\frac{x}{X_{o}}}=m i_{1}^{0} e^{-\frac{x}{X_{o}}}=\Pi_{1}^{0} e^{-\frac{x}{X_{o}}}= \\
& =2.82 \frac{2 \cdot 7.835 \cdot 10^{-3}}{(69.823)^{3}} e^{-\frac{x}{130.57 \cdot 10^{-3}}}=2.82 \frac{0.224 \cdot 10^{-3}}{(69.823)^{2}} e^{-\frac{x}{130.57 \cdot 10^{-3}}}= \\
& =2.82 \frac{3.208 \cdot 10^{-6}}{69.823} e^{-\frac{x}{130.57 \cdot 10^{-3}}}=2.82 \cdot 4.594 \cdot 10^{-8} e^{-\frac{x}{130.57 \cdot 10^{-3}}}= \\
& =12.96 \cdot 10^{-8} e^{-\frac{x}{130.57 \cdot 10^{-3}}}
\end{aligned}
$$

As it results from the equation (2.13), the unit of momentum $P_{z}$ of tool is $\mathrm{kg} \cdot \mathrm{m} \cdot \mathrm{s}^{-1}$ or $\mathrm{N} \cdot \mathrm{s}$, which results from multiplying of the numerator and denominator of the quotient of units by the second, that is s. These operations are as follows

$$
\left[P_{z}\right]_{S I}=\frac{\mathrm{kg} \cdot \mathrm{m}}{\mathrm{s}}=\frac{\mathrm{kg} \cdot \mathrm{m}}{\mathrm{s}} \cdot \frac{\mathrm{s}}{\mathrm{s}}=\frac{\mathrm{kg} \cdot \mathrm{m}}{\mathrm{s}^{2}}=\mathrm{N} \cdot \mathrm{s}
$$

The unit of inertia force $B_{z}$ is $\mathrm{kg} \cdot \mathrm{m} \cdot \mathrm{s}^{-2}$, that results from the equation (2.14). Naturally, it corresponds with newton $(\mathrm{N})$, as 


$$
\left[B_{z}\right]_{S I}=k g \cdot \frac{m}{s^{2}}=N
$$

The time-effect $\Pi_{z}$ has the unit $\mathrm{kg} \cdot \mathrm{m} \cdot \mathrm{s}^{-3}$, that results from the equation (2.15). It may be substituted by the unit $\mathrm{N} \cdot \mathrm{s}^{-1}$, which is the result of the following operation

$$
\left[\Pi_{z}\right]_{S I}=\mathrm{kg} \cdot \frac{\mathrm{m}}{\mathrm{s}^{3}}=\frac{\mathrm{kg} \cdot \mathrm{m}}{\mathrm{s}^{2}} \cdot \frac{1}{\mathrm{~s}}=N \cdot \mathrm{s}^{-1}
$$

Quantitative dependences $P_{z}, B_{z}, \Pi_{z}$ on time $t$ and horizontal coordinate $x$ have been illustrated graphically in Figures 1, 2 and 3, respectively.

$$
\begin{array}{cc}
\frac{p_{z}}{10^{-4} \mathrm{~N} \cdot \mathrm{s}} & \mathrm{p}_{\mathrm{z}}=6.32 \cdot 10^{-4} \mathrm{e}^{-\frac{\mathrm{t}}{69.823}} \\
\mathrm{p}_{\mathrm{z}}=6.32 \cdot 10^{-4} \mathrm{e}^{-\frac{\mathrm{x}}{130.57 \cdot 10^{-3}}}
\end{array}
$$

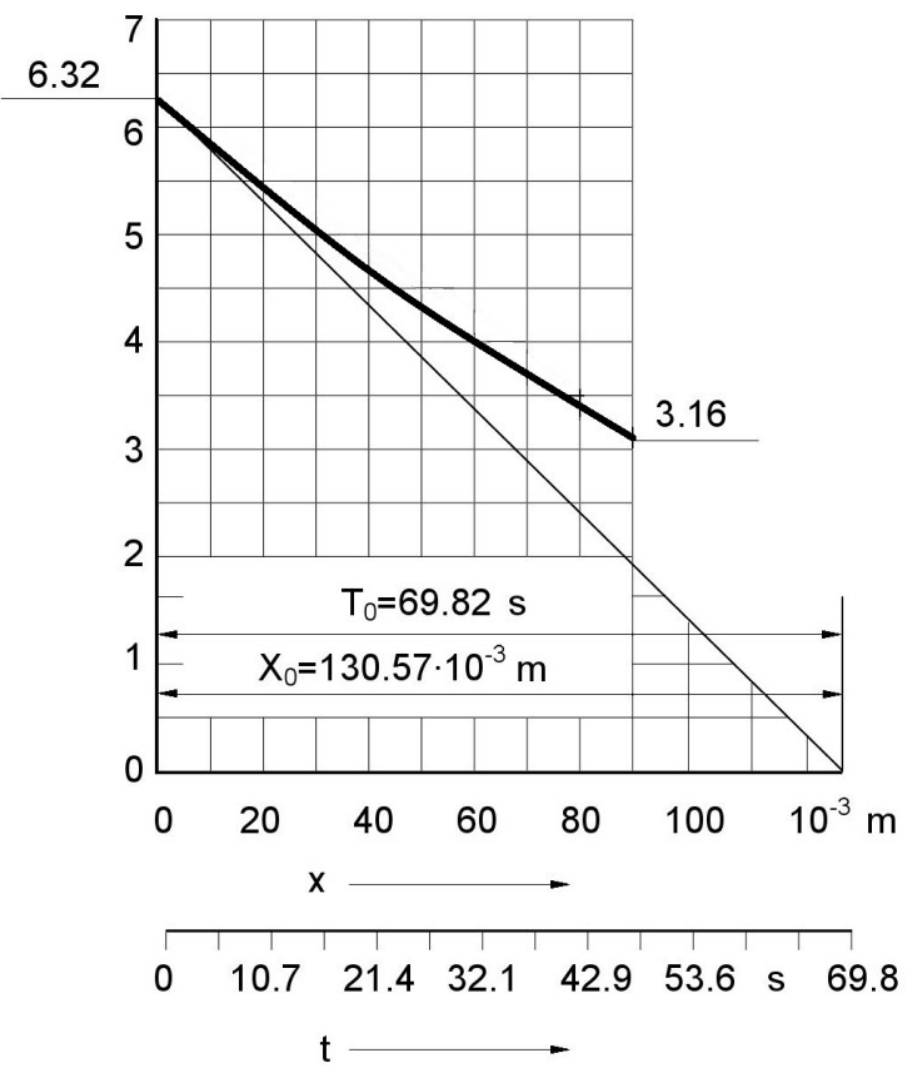

Fig. 1. Dependence of momentum $P_{z}$ of tool on time $t$ and horizontal coordinate $x$ of its position on the path of material treatment.

Here also the right-angled triangles are introduced, with their hypotenuses placed on the directions tangent to the curves in the initial points. The horizontal legs, corresponding with the path constant $X_{o}$ and time constant $T_{o}$, are alike and invariable. 
The vertical legs, corresponding with the initial values of determined magnitudes, are respectively different.

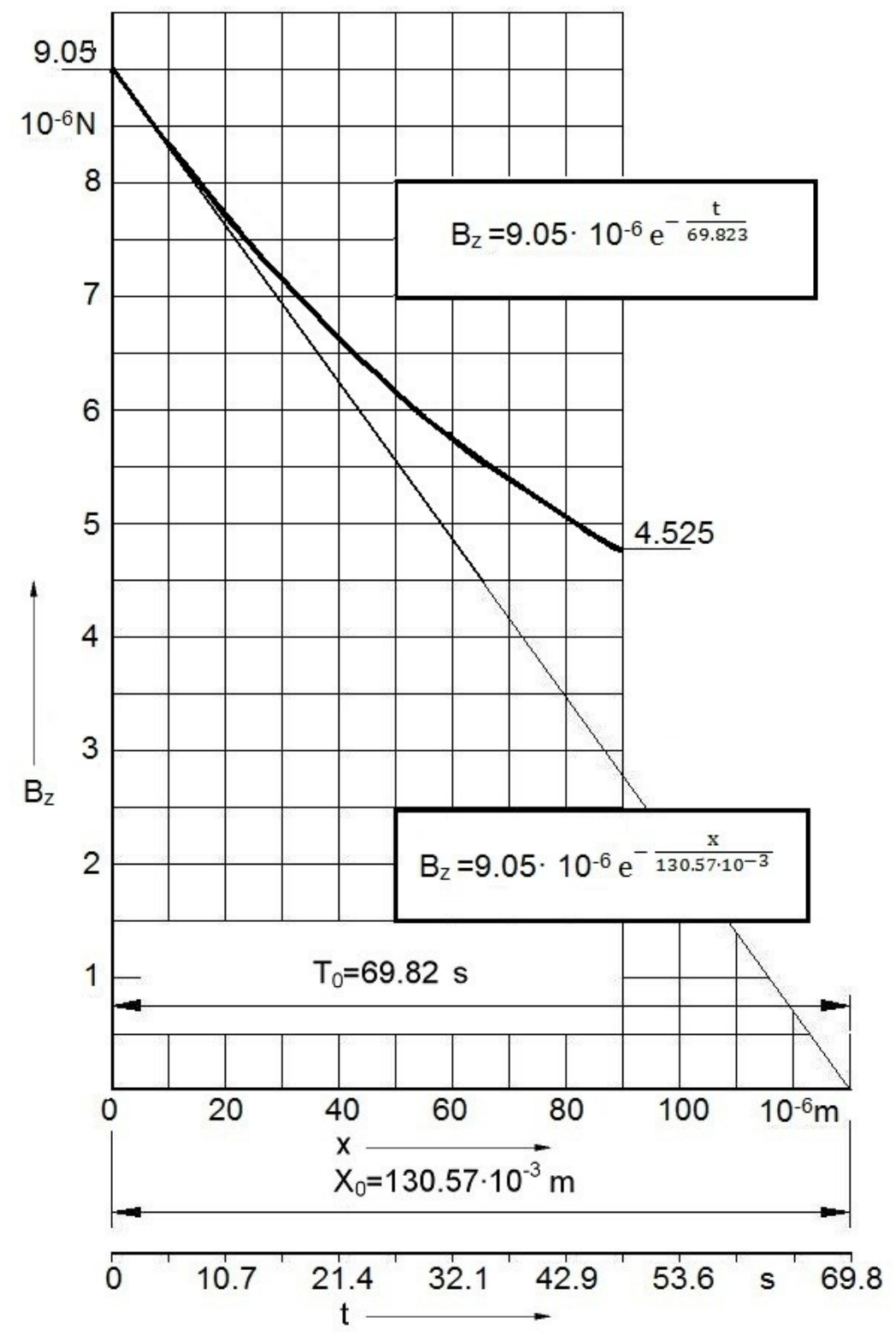

Fig. 2. Dependence of inertia force $B_{z}$ of tool on time $t$ and horizontal coordinate $x$ of its position on the path of material treatment.

Described above the magnitudes characterized the tool dynamics. Now it is worth presenting dynamic characteristics of the whole machining system by determining forces being the measures of particular reasons for material cutting. 


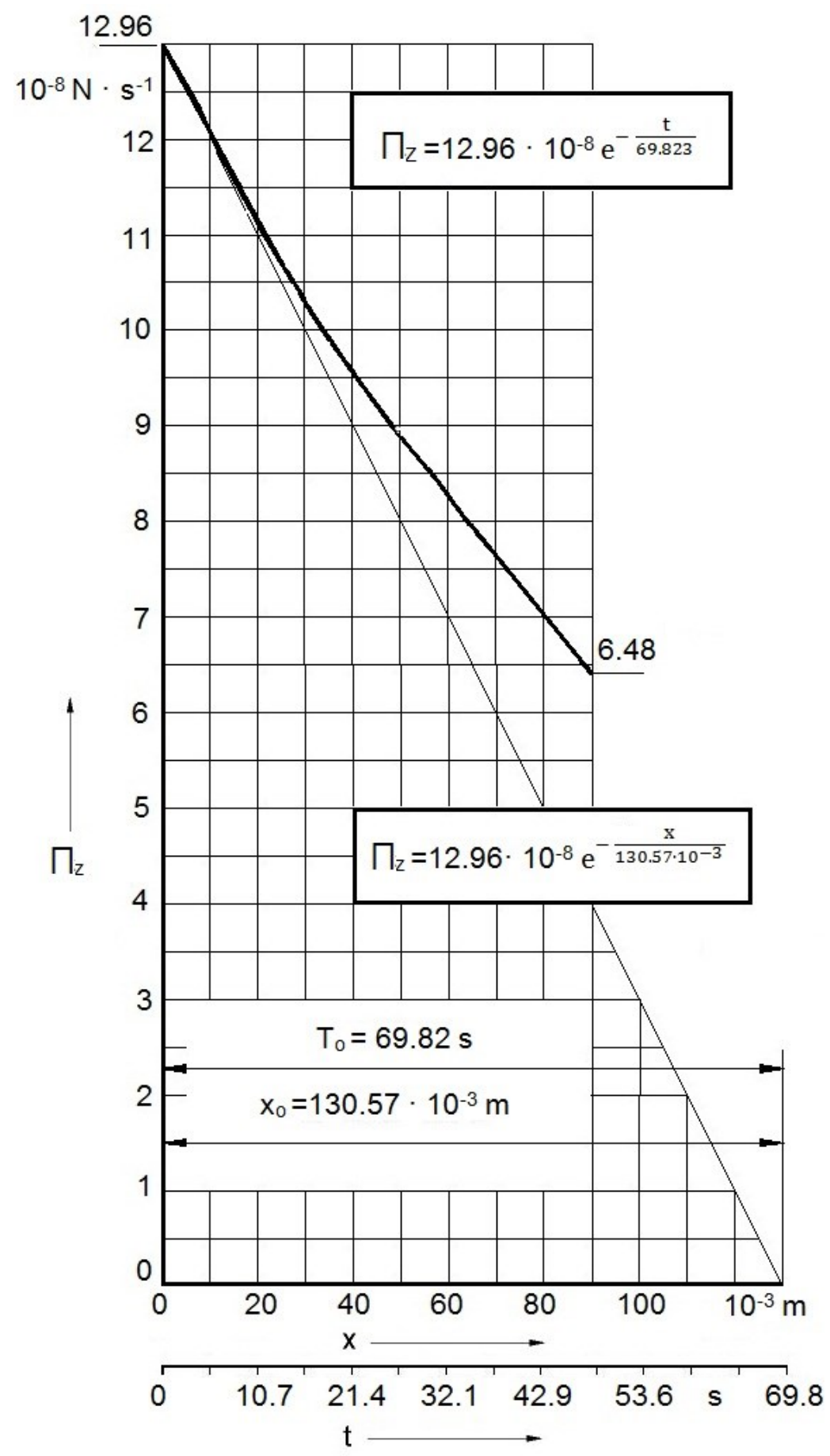

Fig. 3. Dependence of time-effect $\Pi_{z}$ of tool on time $t$ and horizontal coordinate $x$ of its position on the path of material treatment.

The reasons are: gravity, inertia, and repel. Gravity is the ability of attracting body by Earth; inertia is the mass proper resistance of tool; repel means the material action on tool that action results in displacement of the tool. Therefore they will be the forces of: gravity $Q$, inertia $B_{z}$, and repel $R_{z}$. The scheme of material cutting, with the actions of all the reasons, is presented in Fig. 4a, and the measures of these reasons, just forces, are presented in Fig. 4 b. 


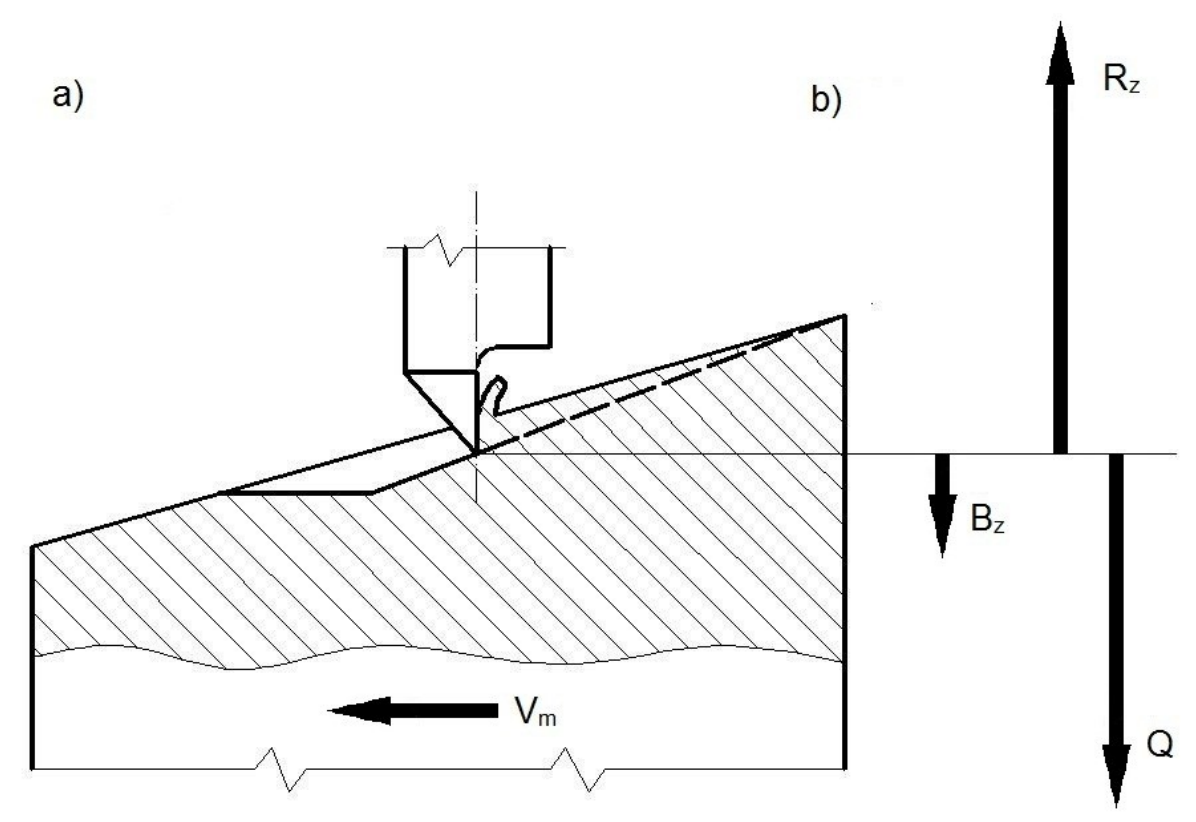

Fig. 4. Scheme of material cutting (a) and the system (b) of force vectors of: inertia $B_{z}$, gravity $Q$, and repel $R_{z}$

The gravity force is the product of mass $m$ and terrestrial acceleration $g$, that is

$$
Q=m \cdot g
$$

The inertia force $B_{z}$ is described by equations (2.5) and (2.11), where the independent variable is time $t$ and horizontal coordinate $x$ of the position of tool on the path of material treatment.

The repel force $R_{z}$ is the sum of both higher given forces, then

$$
R_{z}=B_{z}+Q
$$

Therefore

$$
R_{z}=m \frac{2 z^{*}}{\left(T_{o}\right)^{2}} e^{-\frac{t}{T_{o}}}+m g=m B_{1}^{0} e^{-\frac{t}{T_{o}}}+m g
$$

or

$$
R_{z}=m \frac{2 z^{*}}{\left(T_{o}\right)^{2}} e^{-\frac{x}{X_{o}}}+m g=m B_{1}^{0} e^{-\frac{x}{X_{o}}}+m g
$$

By substituting values of the magnitudes $m, z^{*}, T_{o}, g, X_{o}$ (that are $m=2.82 \mathrm{~kg}$; $z^{*}=7.835 \cdot 10^{-3} \mathrm{~m} ; T_{o}=69.823 \mathrm{~s} ; g=9.81 \mathrm{~m} \cdot \mathrm{s}^{-2} ; X_{o}=130.57 \cdot 10^{-3} \mathrm{~m}$, respectively), to the formulae (2.21) and (2.22), one obtains 


$$
\begin{gathered}
R_{z}=2.82 \frac{2 \cdot 7.835 \cdot 10^{-3}}{(69.823)^{2}} e^{-\frac{t}{69.823}}+2.82 \cdot 9.81=9.05 \cdot 10^{-6} e^{-\frac{t}{69.823}}+27.66 \\
R_{z}=2.82 \frac{2 \cdot 7.835 \cdot 10^{-3}}{(69.823)^{2}} e^{-\frac{x}{130.57 \cdot 10^{-3}}}+2.82 \cdot 9.81=9.05 \cdot 10^{-6} e^{-\frac{x}{130.57 \cdot 10^{-3}}}+27.66
\end{gathered}
$$

Geometric images of force dependences of: repel $R_{z}$, gravity $Q$, and inertia $B_{z}$, on time $t$ and horizontal coordinate $x$ of the tool position on the path of material treatment, have been presented in Fig. 5. As can be seen, the central part of the uniform coordinate system network was left aside by substituting it by a double-dashed line. The values of inertia force are very small, much less than the values of the gravity and repel forces. That means, the cumulative presentation of the dependence plots of all these forces versus variables $t x$ required a special approach. Geometric images of functions are presented in the scale big enough to be clearly visible of the minute changes of the force values.

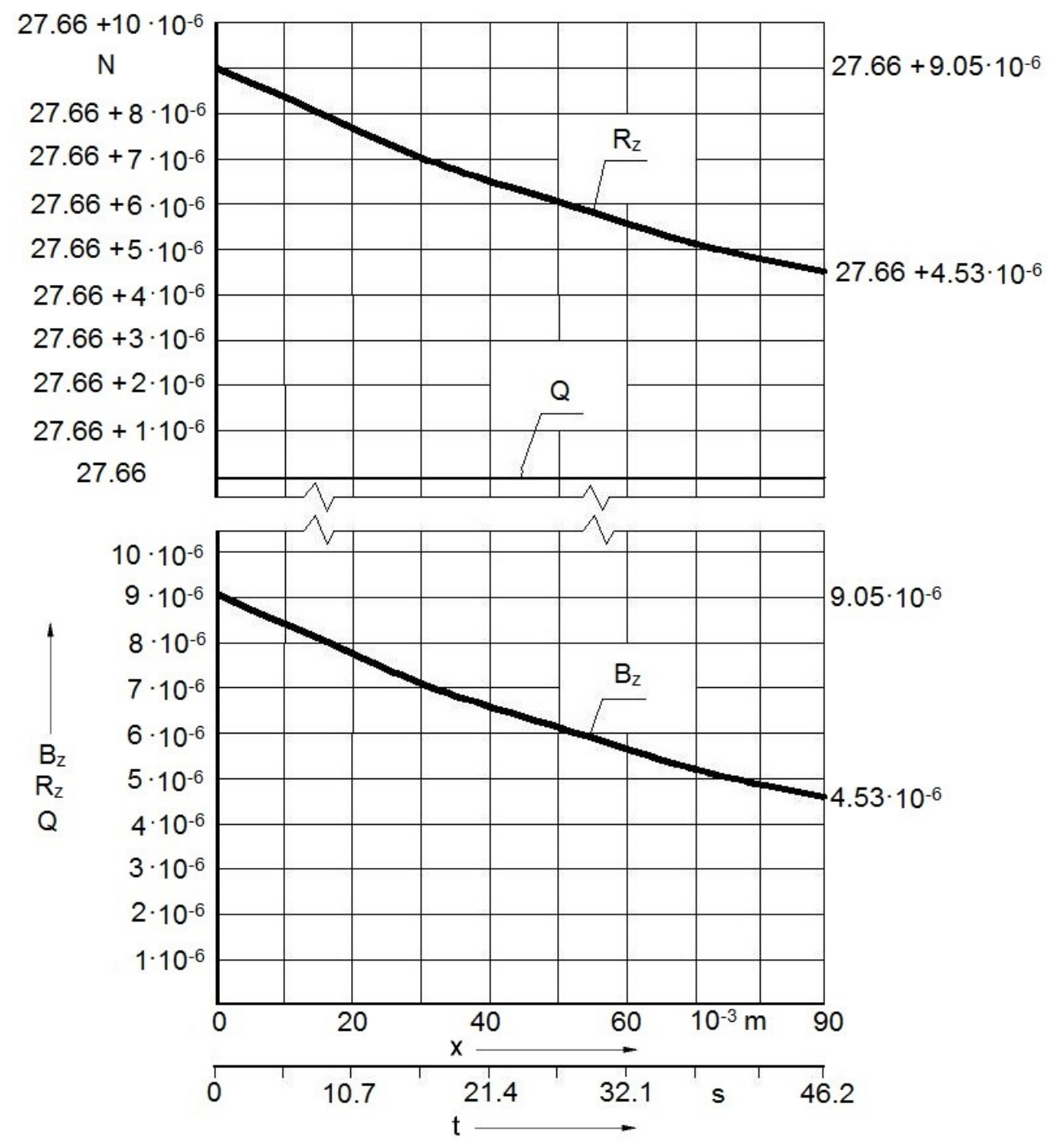

Fig. 5. Dependence of forces: repel $R_{z}$, gravity $Q$, and inertia $B_{z}$, on time $t$ and horizontal coordinate $x$ of the position of tool on the path of material treatment. 


\section{CONCLUSION}

In the summary it is worth underlying the new, quite different method of the procedure in reference to the description of the considered system. That method came into being as a result of very critical look on scheme, i.e. the existent rules in the range of description of dynamics and mechanics of solid.

Finally, it is worth noting that the present work does not close the problem of dynamics and performance of the tool edge fixed flexibly. In the future, the resistance force of tool edge during cutting the material, is to be analyzed and described.

\section{References}

[1] Zdzisław Pluta, Tadeusz Hryniewicz, International Journal of Advanced Manufacturing Technology 62(5) (2012) 529-542; DOI: 10.1007/s00170-011-3813-5

[2] Zdzisław Pluta, Tadeusz Hryniewicz, International Letters of Chemistry, Physics and Astronomy 10(1) (2013) 35-47 (Part 1 - Kinetics).

[3] Zdzisław Pluta, Tadeusz Hryniewicz, International Letters of Chemistry, Physics and Astronomy 4 (2012) 8-16.

[4] Zdzisław Pluta, Tadeusz Hryniewicz, International Letters of Chemistry, Physics and Astronomy 5 (2012) 35-45.

[5] Zdzisław Pluta, Tadeusz Hryniewicz, International Letters of Chemistry, Physics and Astronomy 3 (2013) 67-84.

[6] Zdzisław Pluta, Tadeusz Hryniewicz, International Letters of Chemistry, Physics and Astronomy 7(2) (2013) 85-101.

[7] Zdzisław Pluta, Tadeusz Hryniewicz, Journal of Quantum Information Science (JQIS) 1(3) (2011) 127-134; DOI: 10.4236/jqis.2011.13018

[8] Zdzisław Pluta, Tadeusz Hryniewicz, Journal of Quantum Information Science (JQIS) 1(3) (2011) 149-160; DOI: 10.4236/jqis.2011.13021 